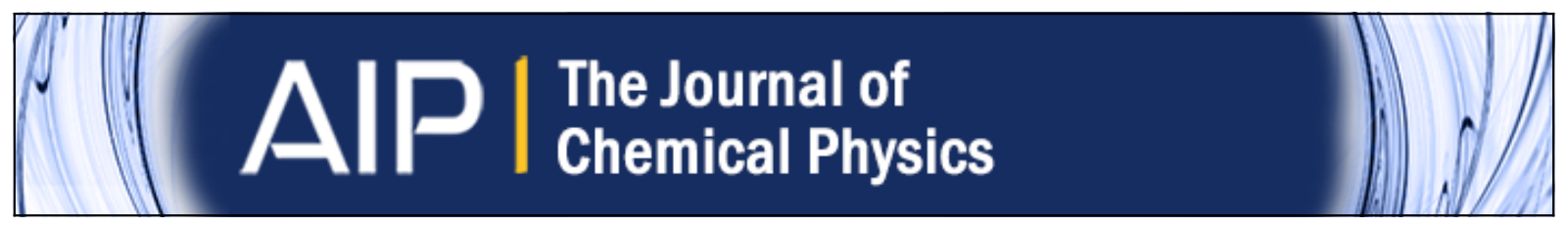

The energy landscape for solvent dynamics in electron transfer reactions: A minimalist model

Y. Tanimura, V. B. P. Leite, and J. N. Onuchic

Citation: The Journal of Chemical Physics 117, 2172 (2002); doi: 10.1063/1.1488588

View online: http://dx.doi.org/10.1063/1.1488588

View Table of Contents: http://scitation.aip.org/content/aip/journal/jcp/117/5?ver=pdfcov

Published by the AIP Publishing

$\underset{\substack{\text { Autloning } \\ \text { puns }}}{A}$ Re-register for Table of Content Alerts 


\title{
The energy landscape for solvent dynamics in electron transfer reactions: A minimalist model
}

\author{
Y. Tanimuraa) \\ Institute for Molecular Science, Myodaiji, Okazaki, Aichi 444-8585, Japan \\ V. B. P. Leite \\ Departamento de Física, IBILCE, Universidade Estadual Paulista, São José do Rio Preto 15054-000, \\ SP, Brazil \\ J. N. Onuchic \\ Department of Physics, University of California, San Diego, La Jolla, California 92093
}

(Received 3 December 2001; accepted 1 May 2002)

\begin{abstract}
Energy fluctuations of a solute molecule embedded in a polar solvent are investigated to depict the energy landscape for solvation dynamics. The system is modeled by a charged molecule surrounded by two layers of solvent dipolar molecules with simple rotational dynamics. Individual solvent molecules are treated as simple dipoles that can point toward or away from the central charge (Ising spins). Single-spin-flip Monte Carlo kinetics simulations are carried out in a two-dimensional lattice for different central charges, radii of outer shell, and temperatures. By analyzing the density of states as a function of energy and temperatures, we have determined the existence of multiple freezing transitions. Each of them can be associated with the freezing of a different layer of the solvent.
\end{abstract}

(C) 2002 American Institute of Physics. [DOI: 10.1063/1.1488588]

\section{INTRODUCTION}

Electron transfer (ET) processes play a central role in several problems in physics, chemistry, and biology. ${ }^{1}$ Most of these reactions occur in condensed phases where the surrounding dielectric medium provides the energetic fluctuations needed in these reactions thereby promoting or hindering thermally activated processes in these systems. Understanding molecular motions and how they determine the reaction coordinate for these ET reactions is, therefore, crucial for a comprehensive description of ET processes.

The early theoretical treatments describing solvation have been based on homogeneous dielectric continuum models that are completely specified by its experimental dielectric dispersion $\epsilon(\omega) .^{2,3}$ In this case, the total solvent polarization around the donor and acceptor sites is used as the ET reaction coordinate. This polarization is usually treated as an effective overdamped harmonic mode. This assumption is equivalent to the use of a harmonic heat bath, which leads to a Gaussian modulation of the energies of the ET donor and acceptor sites. ${ }^{4-7}$ This limit is valid as long as the interactions between an ET system and the solvation molecules have the cumulative effect of a large number of weak interactions, and the central limit theorem comes into play. Treating the solvent as a dielectric continuum provides a reasonable first approximation but it misses the molecular aspects of the solute-solvent interaction. In 1977 Onsager $^{8}$ commented that solvation of a newly created charge (electron) would proceed with the characteristic longitudinal relaxation time $\tau_{L}$ at points far from the charge but, near to it, the

\footnotetext{
a) Author to whom correspondence should be addressed; electronic mail: tanimura@ims.ac.jp
}

response would probably be much slower. This mechanism, which is known as the "inverted snowball" picture, gives a qualitative description of how the solvation dynamics should depart from continuum predictions. Only at points far from the solute, where its field varies slowly compared to the size of the solvent molecules, should the continuum limit of $\tau_{L}$ be attained. This idea served to motivate many of the molecular theories of solvation, which have included some molecular aspects of the solvent. Linearized equilibrium theories of solvation have been extended to correct these dynamic problems; they are known as the dynamical mean-spherical approximations $^{9,10}$ They are molecular models in the sense that the structure imposed by the solute is calculated from an approximated solution of a simple molecular reference system. The real solute/solvent system is mapped onto an idealized hard-sphere solute/dipolar hard sphere solvent. It predicts that at regions close to the cavity (solute), solvent molecules feel the "nonscreened" charge cavity and the response is slower than $\tau_{L}$. In regions far from the cavity (or in the limit the size of the molecules is zero), the continuum limit $\tau_{L}$ is recovered, in agreement with the inverted snowball picture. Simulations have shown that this picture fails ${ }^{11}$ when the linear limit breaks down and, therefore, the overall behavior becomes strongly dependent on the coupling between dipoles and the charge at the cavity. This is the situation investigated in this paper.

Another approach for constructing molecular theories of solvation shifts attention away from the structure created by the solute to the structure and dynamics of the pure solvent alone. One assumes that the most important molecular aspect of solvation is not that correlations between solvent molecules are substantially perturbed by the solute, but that the solute electrostatic field probes the solvent response on 
length scales where molecular aspects of solvent-solvent correlations are important. This response is investigated by studying the solvent density function, with a kinetic description for the density following a generalized Smoluchowski equation. ${ }^{12-14}$ All these models are extensions of the continuum model, where the discreteness (molecular aspect) is included indirectly. Molecular theories that include equilibrium and nonequilibrium solvation processes have been presented. ${ }^{15}$ They are based on renormalized response approaches that incorporate nonlinear aspects of solvation dynamics. They are all based in linear response assumptions, thus they do not include the solvent saturation effects as well as the complexity of multidimensional potential surfaces with multiple minima.

Recent developments of ultrafast nonlinear spectroscopy have made it possible to observe the dynamical aspects of ET processes more directly. ${ }^{16-18}$ Walker et al. ${ }^{19}$ and Tominaga et al. ${ }^{20}$ for example, have investigated the reverse ET, after photoexcitation of the charge transfer band, by monitoring the recovery of the ground state absorption. Kobayashi and co-workers studied the intermolecular ET between excited dye molecules and the electron-donating solvent. ${ }^{21} \mathrm{~A}$ variety of experiments has been carried out and some results clearly indicate deviations from continuum and linear theories. In molecular dynamics studies, multiphasic relaxation regimes with nonexponential relaxation times have also been observed. ${ }^{22}$ The temperature dependence effects are particularly interesting. At high temperatures, where the thermal fluctuation energy is larger than the interaction energies between solvation molecules, the system follows normal diffusion dynamics described by the linear response picture of Born-Marcus theory. ${ }^{1}$ As discussed by Maroncelli et al., however, some ET systems exhibit deviations from this regime. ${ }^{23,24}$ At low temperatures, experimental results for alcohols deviate dramatically from continuum predictions, ${ }^{22}$ suggesting a "glassy" (solvent freezing) behavior. One interesting question is to understand the conditions under which the solvent dynamics can be represented by a single collective reaction coordinate or when these freezing mechanisms cannot be neglected. When this one-dimensional representation is valid, one recovers the successful Born-Marcus theory. Several other computational studies have confirmed the power of this approximation. ${ }^{25}$

The use of all-atom solvent model to discuss these issues is computationally too expensive and it complicates the understanding of the problem. One alternative is to use minimalist models for the solvent, i.e., simpler models that include the necessary complexity to address this question. Onuchic and Wolynes (OW) introduces a minimalist model ${ }^{26}$ for a polar solvent interacting with a charged cavity representing the donor or acceptor site for ET. Although this model is far from representing details of real solvents, it includes the basic features of a rough-energy landscape: multidimensional degrees of freedom, with each solvent molecule being treated independently; a disordered energy landscape with multiple minima; and a polarizable medium around a charged cavity. This model is much simpler than a Brownian dipole lattice model, where the solvent is represented by a rigid cubic lattice of permanent dipole,,${ }^{11,27}$ but it can account for the local structure or frustration of the solvent molecules. Above the "thermodynamic glass" (freezing) transition, it recovers the continuum dielectric limit. Recently the dynamics of the OW model was studied by Leite and Onuchic ${ }^{28}$ which have shown that, at high temperatures, the system exhibits an effective diffusive one-dimensional dynamics, where the Born-Marcus limit is recovered. At low temperatures, a polarization-dependent glassy phase appears and a slow non-self-averaging dynamics is observed. The OW model uses the random energy model approximation ${ }^{29}$ to evaluate the solvent energies, which assumes the solvent energies as random variables. Such an assumption does not include energy correlation between states. Leite recently adapted the OW model to include these correlations. ${ }^{30} \mathrm{~A}$ single shell of solvent molecules around a cavity is described by a two-dimensional system with periodic boundary conditions with nearest-neighbor interaction. There are two main limitations in these approaches. Even when a model is adapted to include energy correlations, the interactions are introduced in a rather nonrealistic way, i.e., the dipoledipole and charge-dipole interactions are just random variables, and are far from representing the actual interactions. These models also deal with a single shell of solvent dipoles around a cavity. The generalization of this model to correct for these two factors is the subject of this paper. Further investigations into determining when a single collective reaction coordinate description is appropriate are also performed.

\section{A MINIMALIST MODEL FOR THE SOLVENT}

A generalization of the model proposed by Onuchic and Wolynes and kinetically explored by Leite and Onuchic is developed. ${ }^{26,28,30}$ The new features are the inclusion of multiple layers of solvent and a more realistic representation of the dipole-dipole and charge-dipole interactions. The OW model considers a single shell of solvent molecules with simple rotational dynamics, represented by dipoles pointing only in two directions, inward and outward, i.e., as Ising spins. In the present work a second layer of dipoles is introduced, and also the positions of the dipoles in both layers include some structural disorder. Figure 1 shows a schematic representation of this model, which is carefully described in Sec. III.

Although individual solvent molecules are treated as simple effective spins, this model is already able to incorporate several of the main features of how polar solvents couple to electron transfer reactions. This kind of minimalist model has been successful in the physics of magnetic systems and, more recently, on problems of protein dynamics and folding; however, very little has been done in terms of exploiting them for realistic solvent effects. Most of the studies to date have been done for continuum models or for very small systems at a full all-atom representation level. Similarly to our recent success in protein folding, ${ }^{31}$ such models are expected to play a central role in establishing the molecular mechanism for polar solvent mediation in electron transfer reactions. These models will also act as bridges between the continuum and the all-atom solvent representations. Note that in this paper we limit our analysis to ET problem, but 


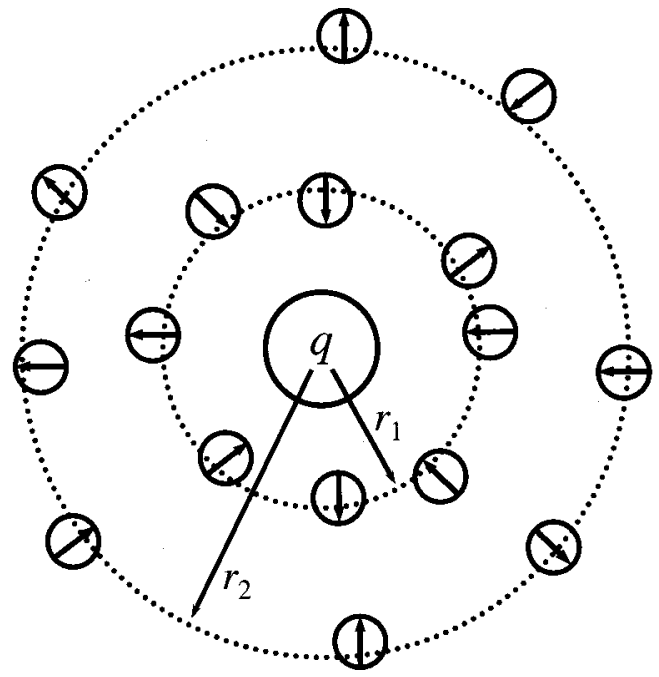

FIG. 1. Schematic view of the two-layer solvent model system. A solute molecule is represented by a point charge in the central cavity. Solvent molecules are represented by two layers of dipoles around the solute in lattice with some spatial disorder. Individual dipoles point only in two directions, inward and outward, and are treated as simple Ising spins.

our study is closely related to nonlinear spectroscopy of a molecular system in the condensed phases. For example, a similar model has been used to account for energy fluctuations of impurity molecules in a glassy environment ${ }^{32,33}$ as the content of single molecule detection, in which the spectral properties of individual impurity molecules in an environment are measured with the ensemble average removed. ${ }^{34}$

Since the initial OW model was based on the random energy model to evaluate the solvent energy, it allowed for a single glass-like transition temperature. In the generalization of this model presented here, we can identify multiple glass (freezing) transitions and are able to associate each of them with the freezing of a different layer of the solvent. It is important to observe how the effect of a central charge completely changes the solvation effect. In order to be able to detect the temperatures for which these layers "freeze" we utilize an approach develop by Socci and collaborators. In the context of protein folding, they defined a useful parameter $Y$, which measures the number of accessible states. ${ }^{35} Y$ reveals the replica symmetry breaking of the structure, and it is a continuous function of order parameters in the folding process, which are the reaction coordinates. In our case, $Y$ (defined in Sec. III) is simplified in just two components, each of them associated to one of the two solvent layers. The use of the $Y$ order parameter allows for a detail exploration of these multiple transitions, which can be associated with the "inverted snowball" picture. To fully explore the physics of the problem, we also study the behavior of several other thermodynamic quantities. The total energy and total polarization are the natural ones, but it is also important to analyze their contributions from each of the individual layers to fully explore the details of the solvent mechanism.

\section{SIMULATION MODEL AND METHODS}

We consider a charged molecule system surrounded by the two layers of solvent molecules with simple rotational dynamics. Individual solvent molecules are represented by dipoles that point in only two directions, inward and outward, and are treated as simple Ising spins. Their positions, unit direction, and strength are represented by $\mathbf{r}_{j}, \mathbf{S}_{j}$, and $\sigma_{j}$, respectively. The charge strength is denoted by $q$. All dipole-charge and dipole-dipole interactions are included in the energy determinations and not simply nearest neighbor interactions. Thus, even under this simple dipole description, the long-range electrostatic contributions are properly incorporated. The interaction energy of solvent is expressed as

$$
E_{\mathrm{solv}}=-\sum_{i=1}^{N} \xi_{i}(q) \sigma_{i}+\sum_{j=2}^{N} \sum_{k=1}^{j-1} J_{j k} \sigma_{j} \sigma_{k}
$$

The charge-dipole interaction and dipole-dipole interaction coefficients are, respectively, expressed as

$$
\begin{aligned}
& \xi_{i}(q)=-\frac{\mu q}{r_{i}^{2}}, \\
& J_{j k}=\mu^{2} \frac{\mathbf{S}_{j} \cdot \mathbf{S}_{k} r_{j k}^{2}-3 \mathbf{S}_{j} \cdot\left(\mathbf{r}_{j k}\right) \mathbf{S}_{k} \cdot\left(\mathbf{r}_{j k}\right)}{r_{j k}^{5}},
\end{aligned}
$$

where $\mathbf{r}_{j k}=\mathbf{r}_{j}-\mathbf{r}_{k}$, and $\mu$ is the dipole moment. Simulations were carried out using typical units with charge measured in terms of 0.1 of the electron charge, dipole factors measured in units of Debyes, and distances measured in units of $2.1 \AA$. Therefore energies are in units of $1.08 \times 10^{-20} \mathrm{~J}$, which is about $2.5\left(k_{B} T\right)$ at room temperature. The values used in the simulations are typical ones for electron transfer in polar solvents. For example, in a case of water solvent, $\mu$ is about 2 and $r_{j} k$ is about 2-3 $\AA$. Our choices of parameters, however, have the goal of demonstrating the behavior of the model and how it affects the electron transfer reaction but they do not refer to any specific solvent.

In this study we limit our analysis to a two-dimensional solvent, although computationally there is no essential difficult in generalizing to the three-dimensional case. Two layers of solvent are included. The inner layer consists of $N_{1}$ dipoles and the outer $N_{2}$ ones. Their coordinates are given by

$$
\begin{aligned}
\mathbf{r}_{j}= & r \cos \left(2 \pi j / N_{1}\right) \mathbf{x}+r \sin \left(2 \pi j / N_{1}\right) \mathbf{y}+\delta \mathbf{r}_{j} \\
& \left(1 \leqslant j \leqslant N_{1}\right),
\end{aligned}
$$

and

$$
\begin{aligned}
\mathbf{r}_{j}= & 2 r \cos \left(2 \pi j / N_{2}\right) \mathbf{x}+2 r \sin \left(2 \pi j / N_{2}\right) \mathbf{y}+\delta \mathbf{r}_{j} \\
& \left(N_{1}<j \leqslant N_{2}\right),
\end{aligned}
$$

where $\delta \mathbf{r}_{j}$ is the Gaussian random distribution of sites defined by

$$
\begin{aligned}
& \left\langle\delta \mathbf{r}_{j}\right\rangle=0, \\
& \left\langle\left|\delta \mathbf{r}_{j}\right|\right\rangle=\delta r .
\end{aligned}
$$

Because of this spatial disorder, the solvent system exhibits frustration that leads to glass behavior at sufficiently low temperatures. In principle, disorder may come from other sources, such as the local environment for each dipole, but the spatial one is sufficient to illustrate the overall solvent mechanism. In our solvent minimalist model, we have included the same number of dipoles in each of the two layers. 
This simplification presents some quantitative limitations when comparisons to real systems are made since the dipole density differs for the two layers. This situation is more likely to correspond to dipolar molecules in confined geometries ${ }^{36}$ such as water molecules in carbon nanotubes. ${ }^{37}$ This approximation decreases the coupling between dipoles for the outmost layers, but it does not change the coupling with the central charge, which is the main issue in electron transfer processes. The effect of the gradual freezing of the layers is indeed enhanced under this scheme. Although the results should be believed only qualitatively, this simplified model already provides all the physical mechanisms associated with gradual freezing of the solvent under the influence of a charge cavity. The unit directions of dipole moments are given by

$$
\mathbf{S}_{j}=\frac{\mathbf{r}_{j}}{\left|r_{j}\right|} .
$$

We consider the two cases for $\sigma_{j}= \pm 1$, where the sign depends on whether the dipoles are pointing toward or away from the charge. The dynamics of the system is described by single-spin-flip kinetics as typically used in Ising model simulations. The probability $P(\Xi ; t)$ for which the system has a spin configuration $\Xi=\left(\sigma_{1}, \sigma_{2}, \ldots, \sigma_{N}\right)$ at time $t$ obeys the master equation

$\frac{\partial P(\Xi ; t)}{\partial t}=\sum_{i}\left[-W_{i}(\Xi) P(\Xi ; t)+W_{i}\left(F_{i} \Xi\right) P\left(F_{i} \Xi ; t\right)\right]$,

where $F_{i} \Xi$ denotes a spin configuration obtained from $\Xi$ by flipping the $i$ th spin: $F_{i} \Xi=\left(\sigma_{1}, \ldots,-\sigma_{i}, \ldots, \sigma_{N}\right)$ for $\Xi$ $=\left(\sigma_{1}, \ldots, \sigma_{i}, \ldots, \sigma_{N}\right)$. The transition probability per unit time, $W_{i}(\Xi)$, for the $i$ th spin to flip in a configuration $S$, is chosen to be of the Glauber type ${ }^{38-40}$

$$
W_{i} \Xi=\frac{1}{2 \tau}\left[1-\sigma_{i} \tanh \left(E_{i}\right)\right]
$$

with

$$
E_{i}=\frac{E_{\mathrm{solv}}\left(F_{i} \Xi\right)-E_{\mathrm{solv}}(\Xi)}{k_{b} T},
$$

where $E_{\text {solv }}(\Xi)$ denotes the energy for the spin configuration $\Xi$ and $\tau$ represents the time scale for a noninteracting spin to flip. It should be noted that this transition probability satisfies the detailed balance condition

$$
W_{i}(\Xi) P_{\mathrm{eq}}(\Xi)=W_{i}\left(F_{i} \Xi\right) P_{\mathrm{eq}}\left(F_{i} \Xi\right),
$$

where $P_{\text {eq }}(\Xi) \propto \exp \left[E_{\text {solv }}(\Xi)\right]$ is the equilibrium distribution.

In this paper, a standard Monte Carlo method, which uses discrete time steps and updates spins in a random sequence, is applied to generate a sample of the time evolution of the spin configurations, which are described by the master equation [Eq. (9)]. We start by randomly choosing an initial configuration $\left\{\sigma_{i}\right\}$. Dynamic moves are then accomplished by single dipole $\left(\sigma_{i}\right)$ flips utilizing the Monte Carlo rule. Dipoles are chosen by going through their array randomly. The Monte Carlo rule is the following. The change in energy $E_{i}$, produced by the trial move, is computed using Eq. (1), and the transition probability $W_{i}(\Xi)$ is determined by Eq. (10). A random number $z$ is selected in the interval $0<z$ $<1$. Finally, if $\tau W_{i}>z$ the move is accepted, i.e., $\sigma_{i}$ is replaced by the new $\sigma_{i}^{\prime}$. Otherwise the move is rejected and the previous configuration is maintained. In either case, every trial is counted as a dynamic step. The time in the master equation (9) corresponds to a Monte Carlo step (MCS), which is defined by repeating the procedure above $N$ times. A single MCS defines the unit of time of the master equation. Although there are other algorithms for generating sample paths, this procedure realistically describes well the dynamics of Ising environments.

During the simulation run, we save all spin configurations $(\Xi)$ for each time step. We then classify the sequence of data under all possible spin configurations. By evaluating the energy or polarization for each spin configuration, we obtain the density of states as the function of energy or polarization.

\section{NUMERICAL RESULTS}

Monte Carlo simulations were carried out for a system composed of $N_{1}=12$ inner dipoles and $N_{1}=12$ outer ones positioned on a two-dimensional lattice. The central charge and the outer-shell radius were varied. The radius of the inner shell was kept fixed at $r_{1}=1$. The position of the dipoles was disordered in each lattice point with a standard deviation of $\delta r=0.1$. Simulations were performed for a broad range of temperatures. In each run, we have discarded the initial 20000 MCSs for thermal equilibration and then recorded 1250000 MCSs of spin configurations and their respective energies. Since one MCS consists of 24 simulation steps, our sample size is about 30000000 . These simulations are sufficiently large for most of our statistical analysis as it becomes apparent later in this section.

As discussed earlier, in order to determine the glass transition the quantity $Y$, which determines the number of accessible states at a given temperature, is calculated. ${ }^{35}$ This quantity is computed for the configurations including all the solvent as well as for partial configurations that only include the inner or outer dipoles. Their definitions are as follows:

$$
\begin{aligned}
& Y_{1}=\sum_{k} \frac{n_{1}(k)^{2}}{N_{\mathrm{samp}}^{2}}, \\
& Y_{2}=\sum_{k} \frac{n_{2}(k)^{2}}{N_{\mathrm{samp}}^{2}}, \\
& Y_{\mathrm{tot}}=\sum_{k} \frac{n_{\mathrm{tot}}(k)^{2}}{N_{\mathrm{samp}}^{2}},
\end{aligned}
$$

where $n_{1}(k), n_{2}(k)$, and $n_{\text {tot }}(k)$ are the number of steps that the spin configuration visits the labeled state $k$ for the innershell, outer-shell, and total solvent system during a simulation run, respectively. The value of $Y$ is inversely proportional to the number of accessible states. In addition to $Y$, the densities of states as a function of polarization $P=\Sigma \sigma_{j}$ and as a function of energy are also determined. Similarly to $Y$, these quantities are also computed for the inner and outer shell. During this single shell calculations, the interaction 

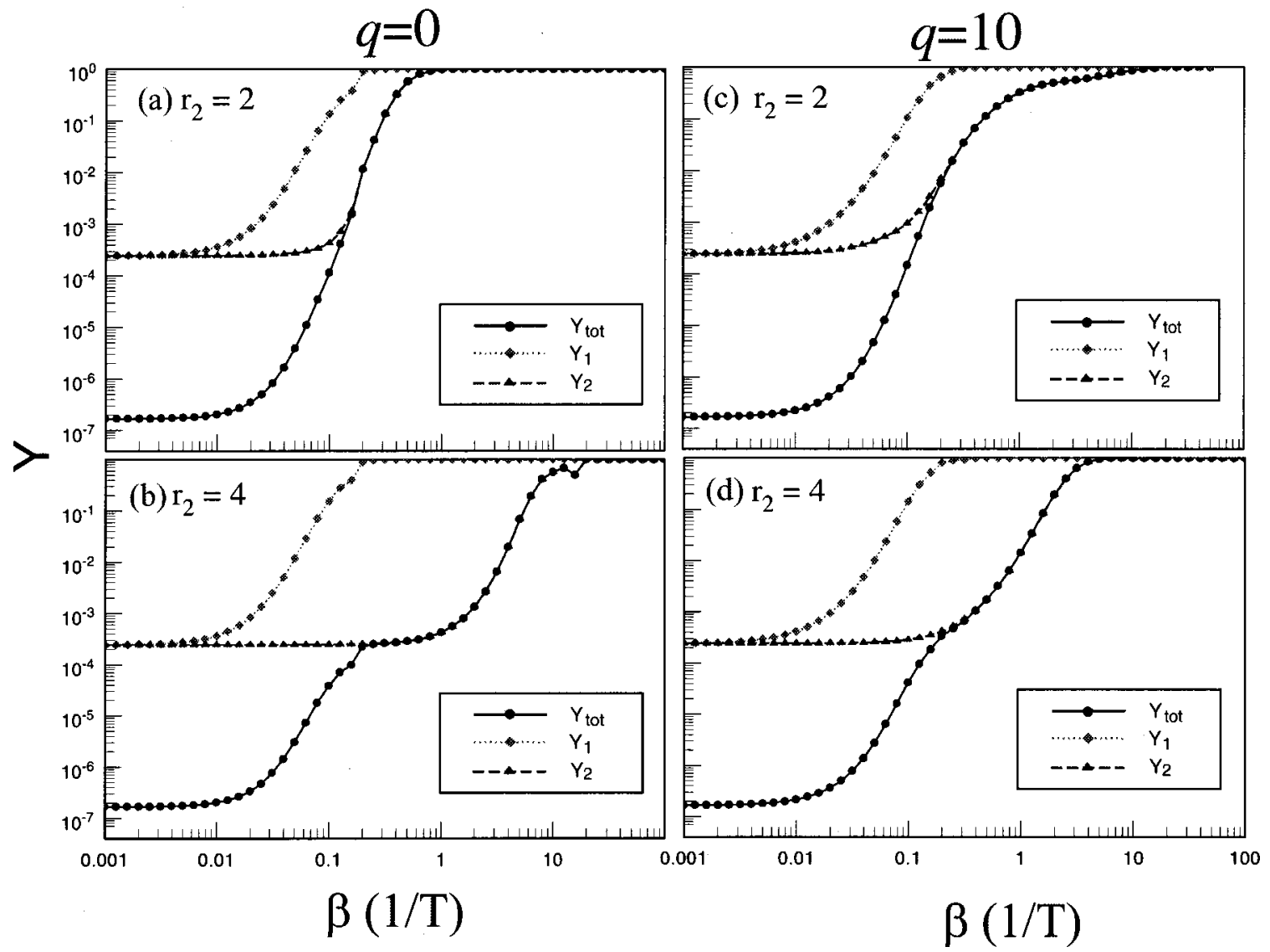

FIG. 2. The function $Y_{1}$ (dotted line), $Y_{2}$ (dashed line), and $Y_{\text {tot }}$ (solid line) calculated from Eqs. (13), (14), and (15) are plotted for different inverse temperatures $(\beta)$ for the cases of: (a) no central charge $(q=0)$ and $r=2$; (b) no central charge and $r_{2}=4$; (c) strong central charge $(q=10)$ and $r_{2}=2$; and finally (d) strong central charge $(q=10)$ and $r_{2}=4$. In the cases of (a) and (c), the total $Y$ shows a single freezing temperature, since the interaction between the inner and outer shell is strong. In the case of $(\mathrm{c})$, since the interaction between the dipoles in the inner shell is stronger than the charge-dipole interaction, $Y_{1}$ for the inner shell shows a similar behavior to the one observed in (a). On the other hand, $Y_{2}$ (outer-shell parameter) is substantially affected by the central charge. In the cases of (b) and (d), the interaction energy between dipoles in the outer shell becomes smaller and the plot for the total $Y$ becomes stepwise, clearly reflecting the independent transitions for the inner and outer shells. In the case of (d), the freezing temperature for $Y_{2}$ occurs at much higher temperatures than the case of (b) due to the influence of the strong central charge.

energy between the inner and outer dipoles is equally shared between the two shells, i.e., half of it assigned to each shell.

Figures 2(a)-2(d) show $Y_{1}$ (dotted line), $Y_{2}$ (dashed lines), and $Y_{\text {tot }}$ (solid line) in the cases of: (a) no central charge $(q=0)$ and $r_{2}=1$; (b) no central charge and $r_{2}=4$; (c) strong central charge $(q=10)$ and $r_{2}=1$; and finally (d) strong central charge $(q=10)$ and $r_{2}=4$ for a broad range of inverse temperatures. In the high temperature limit, simulations estimate $Y \sim 1.6 \times 10^{-7}$. This value is close to the theoretically expected value of $Y=1 / 2^{24} \sim 5.9 \times 10^{-8}$, but it still is slightly large. The origin of this difference is the length of the current simulation, which cannot probe every single state, but the answer is already reasonable. The results for the inner and outer shells are perfect. The number of possible configurations for the inner and outer shells themselves are $N_{\text {shell }}=2^{12}$, and the simulations determine $Y_{1}=Y_{2}=1 / N_{\text {shell }}$ $=2.4 \times 10^{-4}$ for $\beta<0.01$.

First, the situation for a neutral cavity is analyzed. In Fig. 2(a), since the interaction between the inner and outer shell is strong due to the short separation between them, the total $Y$ shows a single "freezing" temperature at $\beta \approx 0.1$. Although the two different shells freeze at different temperatures, as can be observed for the plots for $Y_{1}$ and $Y_{2}$, their respective transitions occur at $\beta \approx 0.06$ and $\beta \sim 0.2$, where the difference between these temperatures is still too small that the two transitions appear to occur in sequence. Therefore it is difficult to separate them from the plot for the total $Y$. For larger values of $r_{2}$, as shown in Fig. 2(b), the interaction energy between dipoles in the outer shell becomes smaller and the shells start to behave more independently. Also the freezing transition temperature of the outer shell reduces substantially compared to the result above. The dynamics of dipoles for different shells are also independent for large $r_{2}$. Therefore the plot for the total $Y$ becomes stepwise, clearly reflecting the independent transitions for the inner and outer shells. This difference is partially due to the fact that the density is different for the two layers, especially in (b), but this additional separation helps with the discussion that follows for the case of a central charge.

The situation becomes more interesting when the central cavity becomes charged. Figures 2(c) and 2(d) are for $q$ $=10$. In the small $r_{2}$ limit, since the interaction between the dipoles in the inner shell is stronger than the charge-dipole interaction, $Y_{1}$ for the inner shell in Fig. 2(c) shows a similar behavior to the one observed in Fig. 2(a). The outer shell, however, is substantially much more affected by the central 


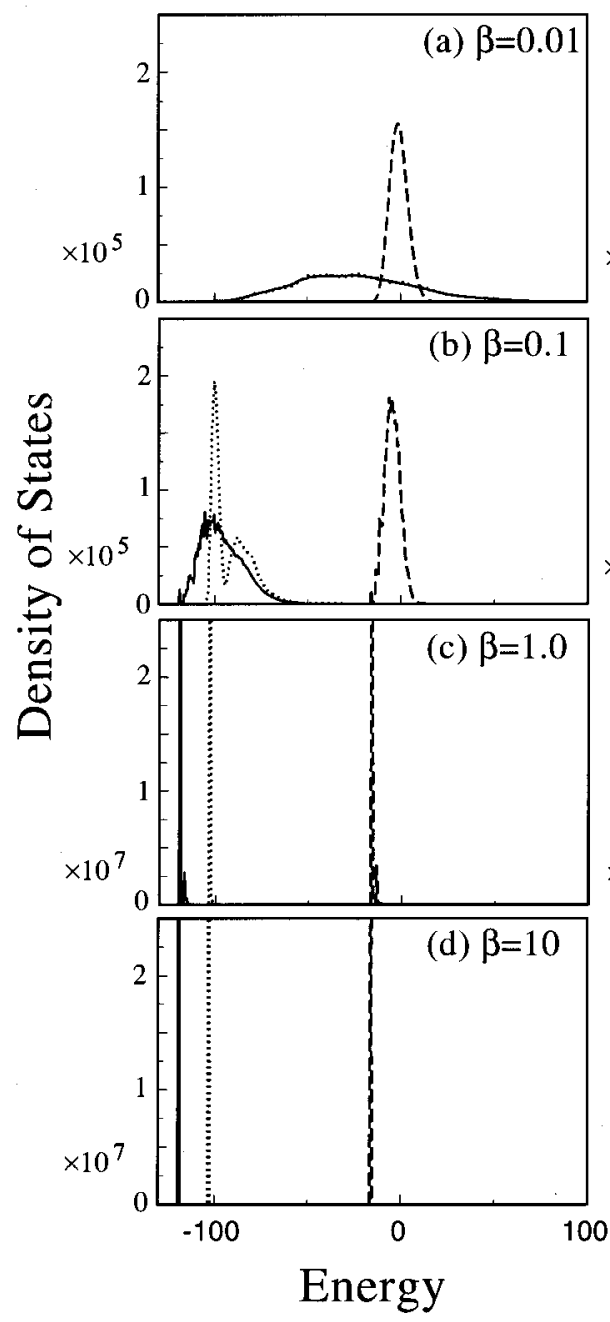

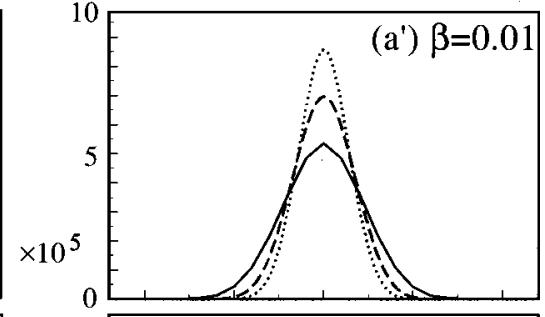
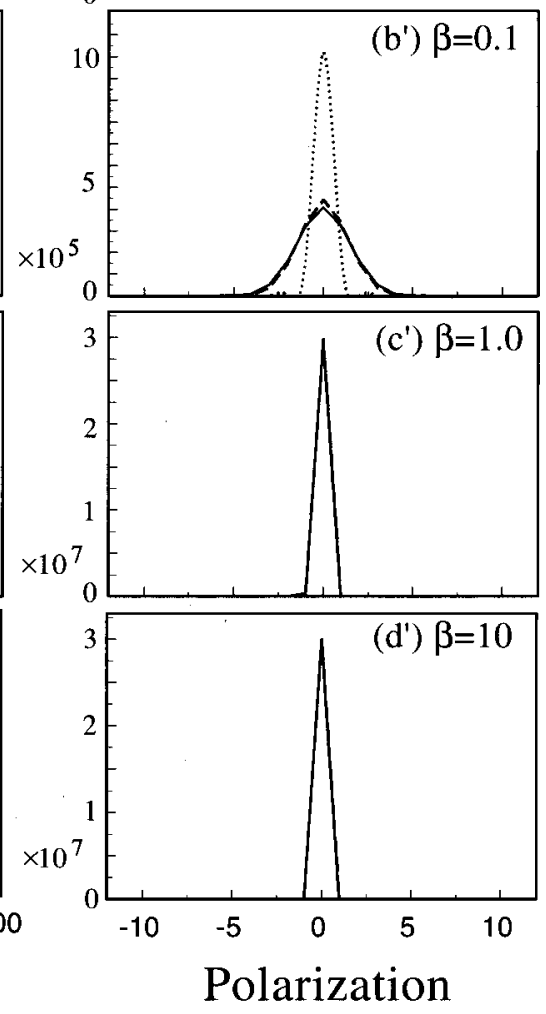

FIG. 3. The density of states as the function of energy $[(a)-(d)]$ and polarization $\left[\left(\mathrm{a}^{\prime}\right)-\left(\mathrm{b}^{\prime}\right)\right]$ in the case of no central charge $(q=0)$ and for $r_{2}=2$ are plotted for different inverse temperatures, $\beta=0.01,0.1,1.0$, and 10 . The dotted, dashed, and solid lines represent the distributions of inner shell, outer shell, and total system, respectively. This figure corresponds to the $Y$ s plotted in Fig. 2(a). In the case of (a)-(d), the difference of the energy scale between the inner and outer shell is observed from the difference of the peak position and width. These distributions become very sharp as the dipoles are frozen. In the case of $\left(a^{\prime}\right)-\left(d^{\prime}\right)$, since no central charge exists in this case, both inner and outer shell distributions are centered around $P=0$. The difference of the energy scale is observed from the difference of the peak width. charge. The freezing temperature and the sharpness of the transition change substantially. In the large $r_{2}$ limit, shown in Fig. 2(d), the energy fluctuations of the outer shell are much larger than for the $q=0$ case [Fig. 2(b)] due to the influence of the strong central charge. Therefore the freezing temperature for this shell occurs at much higher temperatures. $Y_{2}$ in Fig. 2(d) shows freezing for a much smaller $\beta$ than in Fig. 2(b). This transition is also broader, reflecting the wider range of energy fluctuations.

The artificial broader separation between tiers 1 and 2 due to the different dipole density observed in the case of a neutral cavity becomes much smaller. Most of the energetic fluctuations are now determined by the central charge. The fact that the transitions are broader than in (a) and (b) indicates that charge-dipole interactions are now dominant and the gradual freezing of the layers, although still qualitative, is much more realistic.

The microscopic differences discussed above become clear by plotting the density of states as the function of energy and polarization for different temperatures. In Figs. 3 and 4 , these quantities are plotted for the situations with no central charge $(q=0)$ and with strong central charge $(q$ $=10$ ), respectively. For briefness, only the limit of small $r_{2}$ $\left(r_{2}=2\right)$, which corresponds to Figs. 2(a) and 2(c), is analyzed. In these figures, (a)-(d) are the density of states as a function of energy, whereas $\left(\mathrm{a}^{\prime}\right)-\left(\mathrm{d}^{\prime}\right)$ are the density of states as a function of polarization. The inner, outer, and total distributions are plotted in the dotted, dashed, and solid lines, respectively. At high temperatures, both of these figures show energy distributions for both shells that are Gaussian like. This is true for the temperature ranges where $Y_{1}$ and $Y_{2}$ are much smaller than one. This is the regime where the Born-Marcus theory is valid. As the temperature becomes lower, these energy distributions start to deviate from the Gaussian behavior and solvent freezing starts to occur; in Fig. 2, $Y_{1}$ and/or $Y_{2}$ starts to move toward unity.

Figure 3 shows the density of states in the case of no central charge $(q=0)$ and small $r_{2}\left(r_{2}=2\right)$. In Figs. 3(a)$3(d)$, the difference of the energy scale between the inner and outer shell is observed from the difference of the peak position and width. As the temperature is lowered to $\beta>0.01$, the inner shell dipoles start to freeze and their energy distribution shifts to the lower energies, while the distribution of the outer shell dipole remains similar. For $\beta>0.1$ in Fig. 3(b), the outer shell dipoles also start to freeze and their energy distribution shifts to lower energies. The width of both peaks is also sharper in Fig. 3(b), since the number of states occupied at lower energies is much smaller than for higher ones. The situation becomes even more dramatic as the temperature is further reduced [Figs. 3(c) and 3(d)] where freezing of 

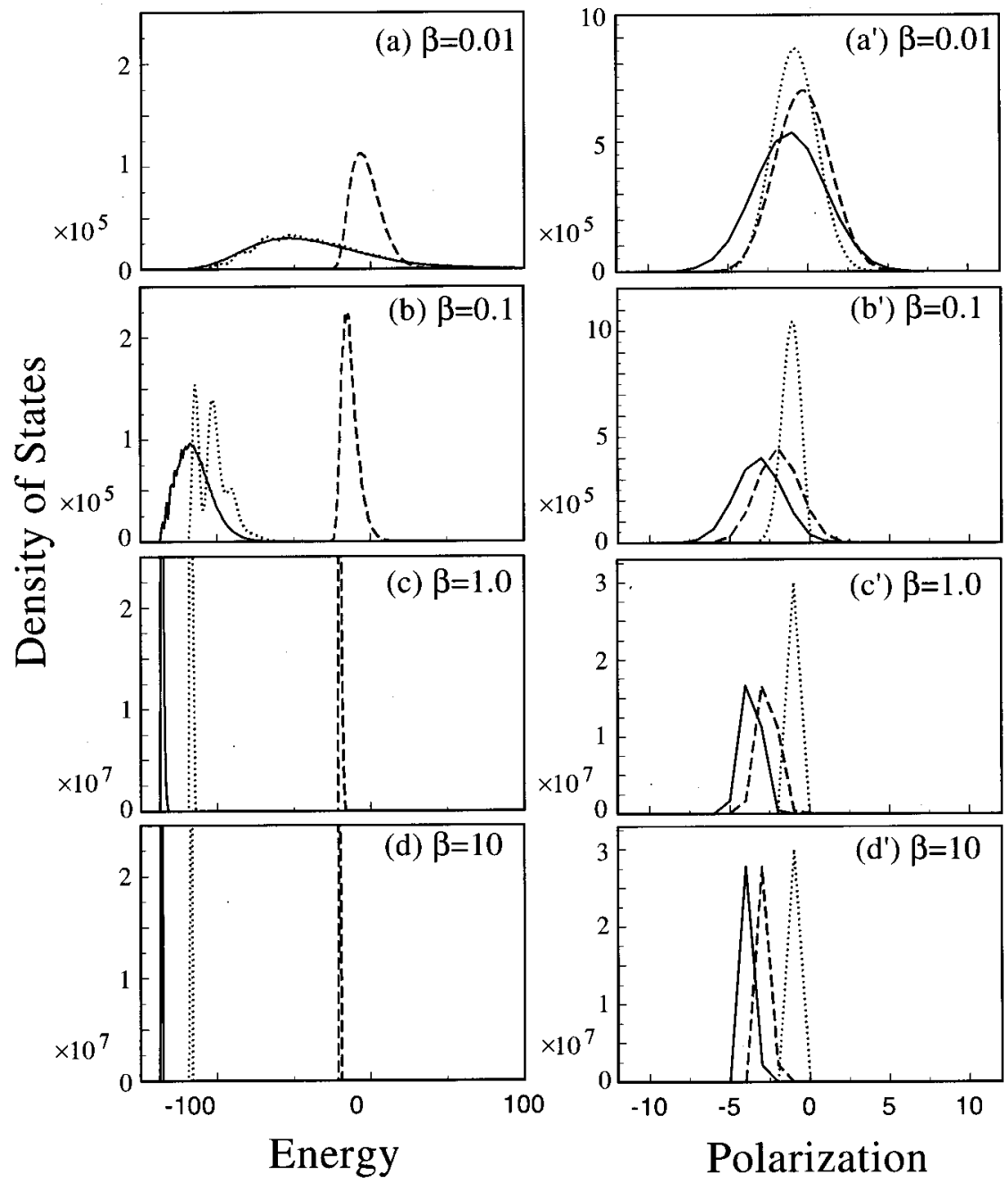

FIG. 4. Similar to Fig. 3 but now for the limit of strong central charge $q$ $=10$. This figure corresponds to the $Y$ s plotted in Fig. 2(c). In the case of (a)-(d), the distributions show a similar behavior to the ones observed in Figs. 3(a)-3(d), since the interaction between the dipoles in the inner shell is stronger than the charge-dipole interaction. These distributions, however, are broader and smoother than those in Fig. 3, since there is the additional energy contribution from the charge-dipole interaction. The interesting new feature is the polarization density of states shown in $\left(a^{\prime}\right)-\left(d^{\prime}\right)$. These polarization distributions are shifted to negative values, especially for the outer shell, because of the interaction with the central charge. both shells becomes apparent. Figures $3\left(a^{\prime}\right)-3\left(d^{\prime}\right)$ display the density of states as the function of polarization. Since no central charge exists in this case, both inner and outer shell distributions are centered around $P=0$. These distributions become very sharp as the dipoles are frozen.

Figure 4 is similar to Fig. 3 but for the case of a central charge $q=10$. The other parameters and the order of figures remain the same. For this small $r_{2}$ case, since the interaction between the dipoles in the inner shell is stronger than the charge-dipole interaction, the probability distribution of the inner shell in Fig. 4(a) shows a similar behavior to the one observed in Fig. 3(a). These distributions, however, are broader and smoother than those in Fig. 3, since there is the additional energy contribution from the charge-dipole interaction. The interesting new feature is the polarization density of states shown in Fig. 4(a')-4(d'). These polarization distributions are shifted to negative values, especially for the outer shell, because of the interaction with the central charge.

\section{CONCLUSIONS}

We have shown that multiple solvent layers can be described by Born-Marcus theory as long as temperatures above the freezing transition are considered. This is the limit where no solvent layer is yet frozen. As the temperature is reduced, freezing starts to occur and the Born-Marcus formula for the solvent polarization starts to break down. The parts of the polar solvent farther from the charge still behave in the Born-Marcus regime but regions closer to it become glassy-like. In the limit that the solvent layers are weakly coupled, different freezing temperatures exist for each of the layers, and the inverted snowball regime is observed. For strongly coupled layers, however, such a simple picture breaks down and a more complex behavior is observed, which is in agreement with what has been observed in some other studies. $^{11}$

This multiple (gradual) transitions could be clearly identified by using a similar approach to ones that we have utilized in protein folding to determine the number of accessible microstates ( $Y$ glass parameter). Although the different dipole densities for the different layers have overemphasized the differences between the two layers, all the qualitative features of the physical mechanisms could be determined with this simple model. Also, in the case of a charged cavity, these quantitative differences are much smaller since most of the energetic fluctuations are determined by charge-dipole interactions. Future extensions of this work will be generalized for three-dimensional representations of the solvent with appropriate molecular densities. 
By utilizing this "replica symmetry breaking" parameter $Y$, we have been able to monitor these multiple freezing transitions and to determine the spatial regions associated with each of them. The next challenge is to utilize models of this kind to quantitatively explain the experimental results that deviate from the linear/continuum models as described in Sec. I of this paper. These studies should allow a direct understanding of the molecular mechanisms by which different polar solvents are coupled to ET reactions. Finally, applications of these models should not be limited to electron transfer; applications to similar problems such as nonlinear optical response should provide us with further understanding of how electronic transitions are mediated by polar solvents.

\section{ACKNOWLEDGMENTS}

J.N.O. is grateful for the hospitality of the Institute for Molecular Science in Japan where part of this work was performed. This work has been supported by the National Science Foundation Grant Nos. MCB-0084797 and INT000419, an international collaboration program with $\mathrm{CNP}_{\mathrm{q}}$, Brazil. V.B.P.L. has been supported by FAPESP, Brazil. Y.T. is grateful for the financial support of a Grant-in-Aid for Scientific Research (B) (Grant No. 12440171) from the Japan Society for the Promotion of Science.

${ }^{1}$ R. A. Marcus, Rev. Mod. Phys. 65, 599 (1993).

${ }^{2}$ R. A. Marcus, J. Chem. Phys. 43, 679 (1965).

${ }^{3}$ B. Bagchi and A. Chandra, Adv. Chem. Phys. 80, 1 (1991).

${ }^{4}$ A. Garg, J. N. Onuchic, and V. Ambegaokar, J. Chem. Phys. 83, 4491 (1985).

${ }^{5}$ J. Jortner and I. Rips, J. Chem. Phys. 87, 2090 (1987).

${ }^{6}$ Y-J. Yan, M. Sparpaglione, and S. Mukamel, J. Phys. Chem. 92, 4842 (1988).

${ }^{7}$ L. E. Fried and S. Mukamel, J. Chem. Phys. 93, 932 (1990).

${ }^{8}$ L. Onsager, Can. J. Chem. 55, 1819 (1977).

${ }^{9}$ P. G. Wolynes, J. Chem. Phys. 86, 5133 (1987).
${ }^{10}$ (a) I. Rips, J. Klafter, and J. Jortner, J. Chem. Phys. 88, 3246 (1988); (b) 89, 4288 (1988).

${ }^{11}$ A. Papazyan and M. Maroncelli, J. Chem. Phys. 98, 6431 (1993).

${ }^{12}$ A. Chandra and B. Bagchi, J. Chem. Phys. 91, 2594 (1989).

${ }^{13}$ B. Bagchi, Annu. Rev. Phys. Chem. 40, 115 (1989).

${ }^{14}$ D. F. Calef and P. G. Wolynes, J. Phys. Chem. 78, 145 (1983).

${ }^{15}$ F. O. Raineri and H. L. Friedman, Adv. Chem. Phys. 107, 81 (1999).

${ }^{16}$ K. Yoshihara, K. Tominaga, and Y. Nagasawa, Bull. Chem. Soc. Jpn. 68, 696 (1995).

${ }^{17}$ P. F. Barbara, T. J. Meyer, and M. A. Ratner, J. Phys. Chem. 100, 13148 (1996).

${ }^{18}$ Y. Tanimura and S. Mukamel, J. Chem. Phys. 101, 3049 (1994).

${ }^{19}$ G. C. Walker, E. Akesson, A. E. Johnson, N. E. Levinger, and P. F. Barbara, J. Phys. Chem. 96, 3728 (1992).

${ }^{20}$ K. Tominaga, D. A. V. Kliner, A. E. Johnson, N. E. Levinger, and P. F. Barbara, J. Chem. Phys. 98, 1228 (1993).

${ }^{21}$ T. Kobayashi, Y. Takagi, H. Kandori, K. Kemnittz, and K. Yoshihara, Chem. Phys. Lett. 180, 416 (1991).

${ }^{22}$ M. Maroncelli, J. Mol. Liq. 57, 1 (1993).

${ }^{23}$ M. Maroncelli and G. R. Fleming, J. Chem. Phys. 89, 5044 (1988).

${ }^{24}$ R. M. Stratt and M. Maroncelli, J. Phys. Chem. 100, 12981 (1996).

${ }^{25}$ A. Warshel and J. K. Hwang, J. Chem. Phys. 84, 4938 (1986).

${ }^{26}$ J. N. Onuchic and P. G. Wolynes, J. Chem. Phys. 98, 2218 (1993).

${ }^{27}$ R. Zwangzig, J. Chem. Phys. 38, 2766 (1963).

${ }^{28}$ V. B. P. Leite and J. N. Onuchic, J. Phys. Chem. 100, 7680 (1996).

${ }^{29}$ (a) B. Derrida, Phys. Rev. B 24, 2613 (1981); (b) Phys. Rev. Lett. 45, 79 (1980).

${ }^{30}$ V. B. P. Leite, J. Chem. Phys. 110, 10067 (1999).

${ }^{31}$ J. N. Onuchic, H. Nymeyer, A. E. Garcia, J. Chahine, and N. D. Socci, Adv. Protein Chem. 53, 87 (2000).

${ }^{32}$ Y. Tanimura, H. Takano, and J. Klafter, J. Chem. Phys. 108, 1851 (1998).

${ }^{33}$ E. Barkai and R. Silbey, J. Phys. Chem. B 104, 342 (2000).

${ }^{34}$ W. P. Ambrose, Th. Basche, and W. E. Moerner, J. Chem. Phys. 95, 7150 (1991).

${ }^{35}$ N. D. Socci, J. N. Onuchic, and P. G. Wolynes, Proteins 32, 136 (1998).

${ }^{36}$ K. Bhattacharyya and B. Bagchi, J. Phys. Chem. A 104, 10603 (2000).

${ }^{37}$ E. Dujardin, T. W. Ebbesen, H. Hiura, and K. Tanigaki, Science 265, 1850 (1994).

${ }^{38}$ R. J. Glauber, J. Math. Phys. 4, 294 (1963).

${ }^{39}$ M. Suzuki and R. Kubo, J. Phys. Soc. Jpn. 24, 51 (1968).

${ }^{40}$ Monte Carlo Methods in Statistical Physics, Topics in Current Physics, edited by K. Binder (Springer, Berlin, 1979), Vol. 1, Chap. 1. 